\title{
The current state of miniature in vivo laparoscopic robotics
}

\author{
Amy C. Lehman • Mark E. Rentschler • \\ Shane M. Farritor · Dmitry Oleynikov
}

Received: 14 November 2006 / Accepted: 18 January 2007 / Published online: 7 February 2007

(C) Springer London 2007

\begin{abstract}
Minimally invasive surgery (MIS) reduces patient trauma and shortens recovery time, but also limits the dexterity of the surgeon because degrees of freedom are lost due to the fulcrum effect of the entry incisions. Visual feedback is also limited by the laparoscope, which typically provides two-dimensional feedback and is constrained by the entry incision. Developments within surgical robotics aim to mitigate these constraints. However, these developments have primarily included large external machines that augment vision and improve dexterity, but are still fundamentally constrained by the use of long tools through small incisions. An alternative concept is the use of miniature in vivo surgical robots that can be placed entirely into the peritoneal cavity through either an abdominal incision, or, after insertion into the stomach through the esophagus, can enter through a gastrotomy. This paper reviews the development of fixed-base camera robots for providing auxiliary views of the surgical field and of mobile robots with a movable platform for vision and task assistance in laparoscopic procedures. Moreover, the progress towards the application of similar robots for natural orifice transluminal endoscopic surgery (NOTES) and forward environments is discussed.
\end{abstract}

A. C. Lehman · M. E. Rentschler · S. M. Farritor

University of Nebraska, N104 Walter Scott Engineering

Center, P.O. Box 880656, Lincoln, NE, 68688-0656, USA

D. Oleynikov ( $\square)$

University of Nebraska Medical Center, 983280 Nebraska Medical Center, Omaha,

NE, 68198-3280, USA

e-mail: doleynik@unmc.edu
Keywords In vivo $\cdot$ Laparoscopy $\cdot$ Robots · Task assistance $\cdot$ Vision

\section{Introduction}

Surgical robotics has become an exciting new field within medicine and surgery over the past decade. A number of devices have been built and used in humans to aide the surgeon or the physician in performing delicate and complex tasks. From robots for orthopedic surgery to robots for general surgery, and all surgical subspecialties in between, the designs have primarily been large externally powered machines that insert end effectors into the human body to accomplish a therapeutic or interventional task. Approximately three years ago, we proposed the use of an active totally intracorporeal miniature surgical device to circumvent the obvious constraints that large complicated machines pose. The main advantage is the ability to externally control a robot that is located completely inside a cavity to accomplish interventional therapeutic goals. In this article we will review the three year history of miniature robotics, what has been shown in animal studies, and how close we are to human trials. We will speculate on future applications of this type of technology and discuss the possibility of surgical intervention that exists with total intracorporeal surgical machines.

\section{Background}

The development of techniques within minimally invasive surgery (MIS) allows surgeons to perform 
procedures through small incisions or natural orifices, thereby offering benefits to the patient including reduced trauma and quicker recovery times. However, the visual and dexterous constraints imposed, including two-dimensional visual feedback and constrained motion, often limits minimally invasive surgeries to less complicated procedures. The use of robotics attempts to alleviate these constraints and further advance MIS.

Commercially available MIS robotics, such as da Vinci (Intuitive Surgical), generally have multiple arms that are teleoperated by the surgeon. These robots attempt to augment surgical dexterity and visual feedback through features including articulating end effectors, tremor filtering, motion reversal correction, stereoscopic vision, and motion scaling [1-3]. Challenges remain in the universal usage of robotic systems for laparoscopic MIS. These robotic systems are implemented from outsidethe body, and therefore remain constrained to some degree by the entry-point incisions. Robotic arms are long and bulky to allow the necessary range of motion for maneuverability of the tools. Moreover, large excursion arcs of the arms result in frequent collisions outside of the patient, and if ports are incorrectly positioned collisions can occur inside the patient as well [1]. The widespread use of these robotics for laparoscopic MIS remains limited due to high cost, large size, and the diminished impact of the dexterous improvements in the performance of lesscomplex laparoscopic procedures.

Applications of robotic technologies for areas outside of laparoscopy are also being explored. Endoscopes with articulating distal tips have been experimentally developed for enhancing maneuverability and vision [4-7]. Accompanying advancements in locomotion techniques based on the inch worm motion scheme or rolling stents [8-12] will potentially lead to the development of a fully autonomous robot for exploring natural orifices. Smaller endoscopic microcapsules with stopping and locomotion capabilities are also being developed to assist in the diagnosis and treatment of gastrointestinal diseases [13]. Finally, developments have been made in cardiac applications for providing a stabilized platform for heart manipulation [14]. Despite the successes, the application of these technologies in laparoscopy remains limited because the problems addressed are fundamentally different than those encountered for surgical procedures within the peritoneal cavity.

\section{Laparoscopic in vivo miniature robotics}

A novel approach to address the visual and dexterous constraints associated with laparoscopic MIS is the use of in vivo miniature robots that fit entirely inside the peritoneal cavity. The robots can be categorized as having either a fixed-base or mobile platform.

\section{Fixed-base robots}

The primary objective of laparoscopic robots with a fixed-base platform is to provide auxiliary viewpoints of the surgical field, thereby augmenting vision and improving depth perception. These robots are placed by the surgeon using traditional laparoscopic tools and can be relocated throughout a procedure without the need for a new incision. Initial work within this area has led to the development of a miniature pan and tilt camera robot $[15,16]$.

The miniature camera was designed and built using only off-the-shelf components. The robot, shown in Fig. 1, rotates the camera about two independent axes, allowing for panning of $360^{\circ}$ and tilting of $\pm 45^{\circ}$. Illumination of the surgical field is accomplished using two light-emitting diodes (LEDs). The platform of this robot consists of legs that are abducted by torsion
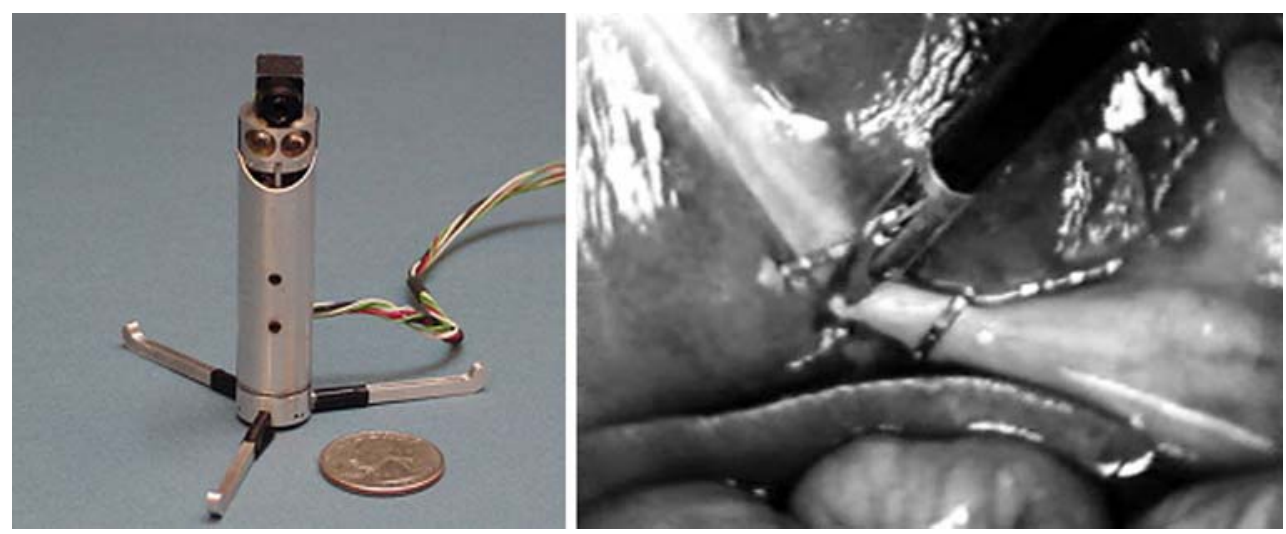

Fig. 1 Pan and tilt camera robot (left) provides visual feedback during cholecystectomy (right) 
springs. The platform design and the $15 \mathrm{~mm}$ diameter housing allows for the insertion of the robot using a standard laparoscopic port.

The pan and tilt camera robot was used in combination with a standard laparoscope to provide video feedback during a porcine cholecystectomy. The additional views provided by this robot aided the surgeon in the planning and placement of trocars and provided views from alternative angles throughout the procedure.

\section{Mobile robots}

Mobile robots provide a remotely controlled movable platform for vision and surgical task assistance. The basic design of a mobile robot consists of two independently driven wheels that provide for forward, reverse, and turning motion. A tail prevents counter-rotation while allowing the robot to reverse directions. Viscoelastic modeling together with bench top and in vivo testing led to the development of a helical wheel design for the mobile robot [17]. A helical wheel design robot with a $15 \mathrm{~mm}$ diameter, as shown in Fig. 2, has proven maneuverable on all of the pelvic organs (liver, spleen, small and large bowel) and capable of climbing highly deformable structures two to three times its height without causing any visual tissue damage in a porcine model.

A similar mobile robot with the addition of an adjustable-focus camera for vision assistance has also been developed. The adjustable-focus capabilities of the camera allows for a greater understanding of depth within the peritoneal cavity and the views provided are comparable to those from currently available laparoscopes. Similar to the fixed-base robots, the mobile adjustable-focus robotic camera aided the surgical team with the planning and vision for additional trocar insertions, and with tool placement during a laparoscopic gallbladder removal in a porcine model, as shown in Fig. 3 [18]. Throughout this procedure, the mobile camera robot provided the sole visual feed-

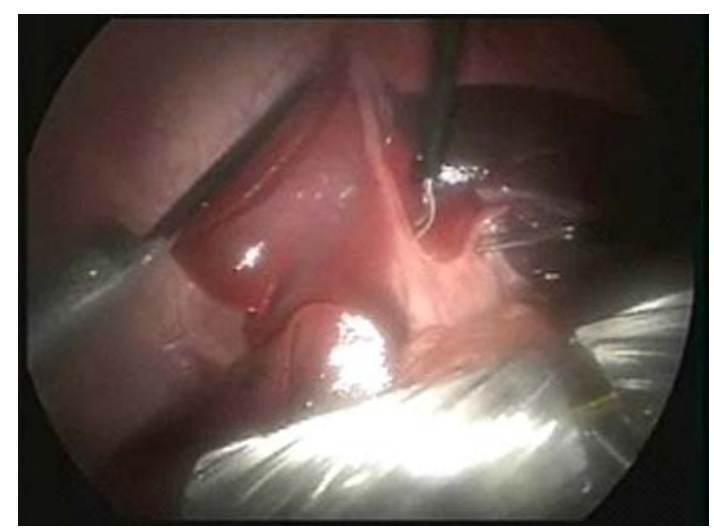

Fig. 3 Mobile camera robot provides visual feedback for cholecystectomy as viewed by laparoscope

back, thereby demonstrating the potential for reducing patient trauma through the removal of the third camera port incision.

The ability to provide task assistance, in addition to the vision assistance previously discussed, has been demonstrated with a biopsy camera robot, as shown in Fig. 4 [19]. This robot is based on the camera system and mobility design of the mobile camera robot. The adaptation of a biopsy forceps device provides for biopsy capabilities. Successful biopsy of three samples of hepatic tissue in a porcine model has been demonstrated. The onboard camera was used to locate an adequate biopsy site as the robot traversed the peritoneal cavity. The robot was then driven using remote control to the chosen site. The grasper was used to cut almost all of the tissue with the remainder being pulled free by driving the robot slowly away from the biopsy site. The tissue sample was then extracted and retrieved. The successful completion of this porcine test demonstrated the capability to perform a one-port laparoscopic biopsy.

The feasibility of the application for in vivo miniature robots for natural orifice transluminal endoscopic surgery (NOTES) procedures has been successfully demonstrated using a mobile robot in a porcine model
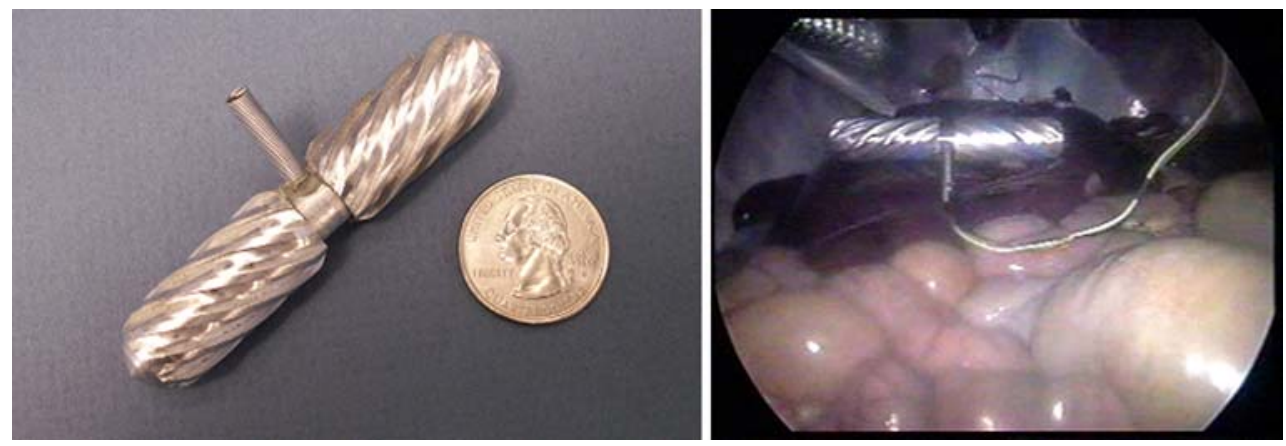

Fig. 2 The $15 \mathrm{~mm}$ mobile robot (left) successfully navigates within the peritoneal cavity (right) 


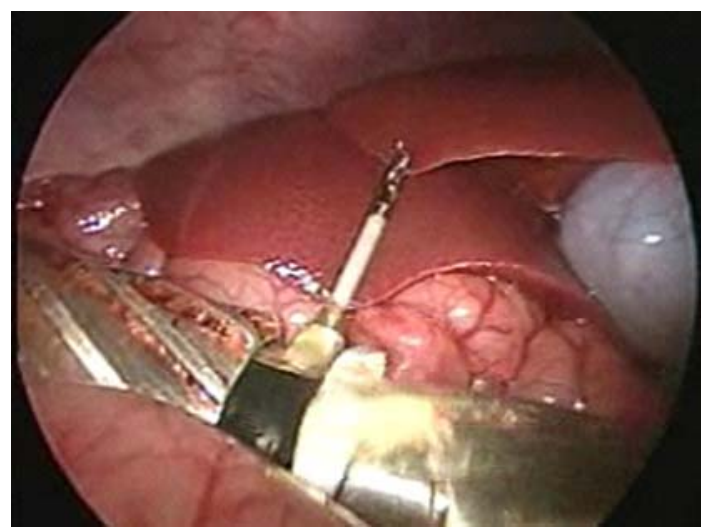

Fig. 4 Mobile biopsy camera robot performs biopsy of hepatic tissue as viewed by laparoscope

[20]. A $12 \mathrm{~mm}$ diameter in vivo mobile robot, as shown in Fig. 5, was able to traverse within the gastric cavity under esaphagogastroduodenoscopic (EGD) control upon insertion using a sterile overtube. Sufficient traction for movement within the gastric cavity without causing visible tissue damage was obtained using the helical wheel design developed for laparoscopic procedures. The robot was then inserted into the peritoneal cavity through a gastrotomy. The ability to traverse the entire peritoneal cavity was demonstrated. For this procedure, the robot was observed using an endoscope. Future work will include the incorporation of onboard cameras, similar to the laparoscopic mobile robots, capable of providing visual feedback for endoluminal procedures. After successfully demonstrating the mobility within the peritoneal cavity, the endoluminal robot was retracted into the gastric cavity and retrieved using an endoscopic snare.

\section{Future applications of miniature robots}

Current work is focused on the continued development of miniature robots for forward environments and NOTES applications. The further development of wireless capabilities and the design of a biopsy grasper capable of clamping a severed artery are important for the application of in vivo robotics as a remote first responder in forward environments such as battlefields. Additional focus is placed on the continued development of miniature robots for NOTES applications to further reduce patient trauma. With the integration of a camera and a manipulator, the endoluminal mobile robot could provide assistance during procedures within the peritoneal cavity. Eventually, minimally invasive procedures will incorporate a team of miniature in vivo robots equipped with sensors and manipulators to cooperatively assist the surgical team from within the gastric or peritoneal cavity. Human trials are being planned for the near future to test these machines and define their role in the future of robotic surgery.

\section{Discussion}

The role of miniature robots in medicine will surely change how we see and treat patients. The technology today exists to deliver a small, agile, remotely controlled machine with the ability to perform simple surgical tasks. Demonstrations have shown the effectiveness of these devices for biopsy, investigation, and as an assist device for complex surgical procedures. It is conceivable that smaller robots with more-complex
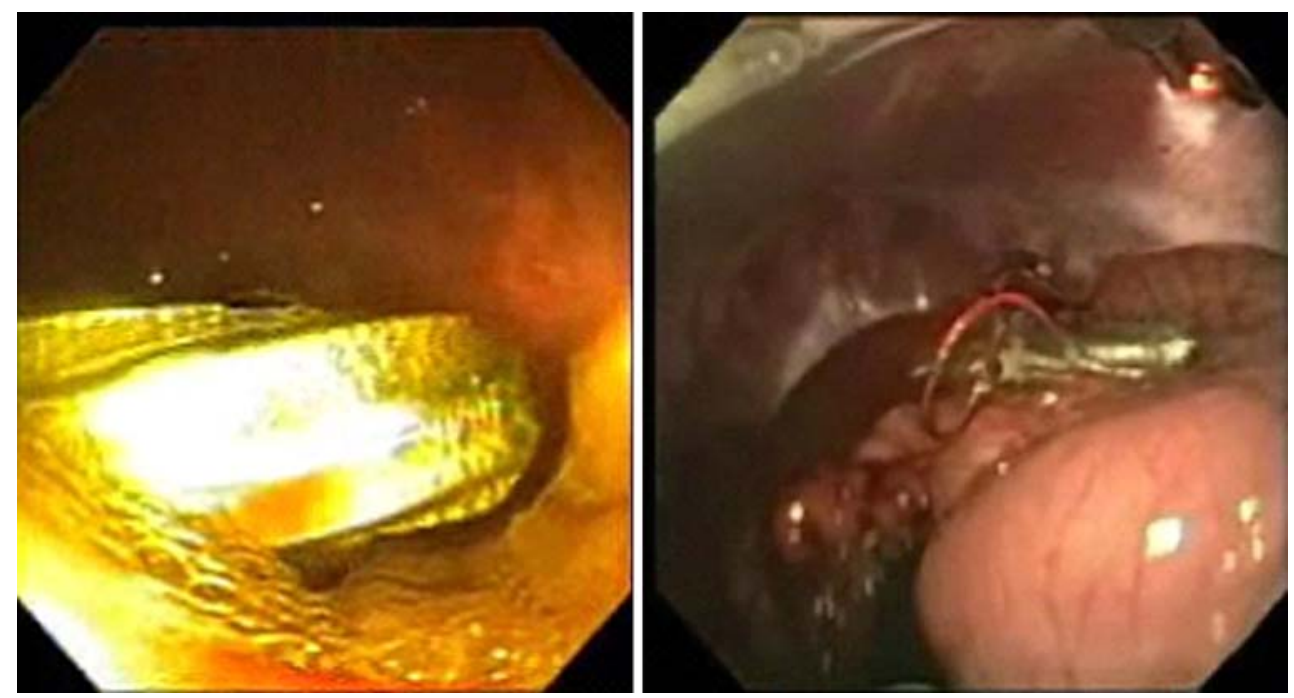

Fig. 5 The $12 \mathrm{~mm}$ mobile robot successfully traverses within the gastric (left) and peritoneal (right) cavities 
control functions will be used in a variety of applications from intravascular to intracranial. Perhaps future robots will even be small enough to be used on a cellular basis. Such machines would be delivered through needles or would be swallowed instead of being inserted through large incisions. These robots will not replace the surgeon who is treating a patient with a large complex tumor. Perhaps what they will do is allow surgeons to investigate patients and remove the tumor when it is at the cellular level. New technology is already allowing earlier detection of diseases and it is important that we develop concomitantly interventions that are less invasive to the patient so we can take full benefit of this early detection.

Robotic surgery is just at the dawn of its development. Many issues remain. Miniaturization of optical components and more-robust batteries are the first few areas that would have to be tackled. Much more human experience will be needed to determine if the increased complexity and costs will be outweighed by greater safety and utility.

\section{References}

1. Ballantyne GH (2002) Robotic surgery, telerobotic surgery, telepresence, and telementoring. Review of early clinical results. Surg Endosc 16(10):1389-1402

2. Corcione F, Esposito C, Cuccurullo D et al. (2005) Advantages and limits of robot-assisted laparoscopic surgery: preliminary experience. Surg Endosc 19(1):117-119

3. Moorthy K, Munz Y, Dosis A et al. (2004) Dexterity enhancement with robotic surgery. Surg Endosc 18(5):790795

4. Ikuta K (1988) The application of micro/miniature mechatronics to medical robotics. Proceedings of the 1988 IEEE international workshop on intelligent robots systems 9-14

5. Dario P, Valleggi R, Pardini M et al. (1991) A miniature device for medical intracavitary intervention. In: Proceedings of the IEEE micro electric mechanical systems 171-175

6. Suzumori K, Iikura S, Tanaka H (1991) Development of flexible microactuator and its applications to robotic mechanisms. In: Proceedings of the 1991 IEEE international conference on robotics and automation 2:1622-1627
7. Fukuda T, Guo S, Kosuage K et al. (1994) Micro active catheter system with multi degrees of freedom. In: Proceedings of the 1994 IEEE international conference on robotics and automation 3:2290-2295

8. Slatkin AB, Burdick J, Grundfest W (1995) The development of a robotic endoscope. In: Proceedings of the 1995 IEEE/ RSJ international conference on intelligent robots and system 95, 'Human Robot Interaction and Cooperative Robots' 2:162-171

9. Phee L, Menciassi A, Gorini G et al. (2002) An innovative locomotion principle for minirobots moving in the gastrointestinal tract. In: Proceedings of the 2002 IEEE international conference on robotics and automation 1125-1130

10. Breedveld P (2006) Development of a rolling stent endoscope. The first IEEE/RAS-EMBS international conference on biomedical robotics and biomechatronics 921-926

11. Dodou D, Girard D, Breedveld P et al. (2005) Intestinal locomotion by means of mucoadhesive films. In: Proceedings of the 12th international conference on advanced robotics 352359

12. Menciassi A, Dario P (2003) Bio-inspired solutions for locomotion in the gastrointestinal tract: background and perspectives. Philos Transact A Math Phys Eng Sci 361(1811):22872298

13. Karagozler ME, Cheung E, Kwon J et al. (2006) Miniature endoscopic capsule robot using biomimetic micr-patterned adhesives. The first IEEE/RAS-EMBS international conference on biomedical robotics and biomechatronics 105-111

14. Patronik N, Zenati MA, Riviere CN (2005) Preliminary evaluation of a mobile robotic device for navigation and intervention on the beating heart. Comput Aided Surg 10(4):225-232

15. Rentschler M, Hadzialic A, Dumpert J et al. (2004) In vivo robots for laparoscopic surgery. Stud Health Technol Inform 98:316-322

16. Oleynikov D, Rentschler M, Hadzialic A et al. (2005) Miniature robots can assist in laparoscopic cholecystectomy. Surg Endosc 19(4):473-476

17. Rentschler M, Dumpert J, Platt S et al. (2006a) Modeling, analysis, and experimental study of in vivo wheeled robotic mobility. IEEE Trans Rob 22(2):308-321

18. Rentschler ME, Dumpert J, Platt SR et al. (2006b) Mobile in vivo camera robots provide sole visual feedback for abdominal exploration and cholecystectomy. Surg Endosc 20(1):135-138

19. Rentschler M, Dumpert J, Platt S et al. (2007) An in vivo mobile robot for surgical vision and task assistance. ASME J Med Devices (in press)

20. Rentschler ME, Dumpert J, Platt SR et al. (2007) Natural orifice surgery with an endoluminal mobile robot. Surg Endosc (in press) 